\title{
EXTENDED QUADRATIC ALGEBRA AND A MODEL OF THE EQUIVARIANT COHOMOLOGY RING OF FLAG VARIETIES
}

\author{
A. N. KIRILLOV AND T. MAENO
}

To Ludwig Dmitrievich Faddeev on the occasion of his 75 th birthday

\begin{abstract}
For a root system of type $A$, a certain extension of the quadratic algebra invented by S. Fomin and the first author is introduced and studied, which makes it possible to construct a model for the equivariant cohomology ring of the corresponding flag variety. As an application, a generalization of the equivariant Pieri rule for double Schubert polynomials is described. For a general finite Coxeter system, an extension of the corresponding Nichols-Woronowicz algebra is constructed. In the case of finite crystallographic Coxeter systems, a construction is presented of an extended Nichols-Woronowicz algebra model for the equivariant cohomology of the corresponding flag variety.
\end{abstract}

\section{§1. INTRODUCTION}

In the paper [6], S. Fomin and the first author introduced and studied a model for the cohomology ring of flag varieties of type $A$ as a commutative subalgebra generated by the so-called Dunkl elements in a certain noncommutative quadratic algebra $\mathcal{E}_{n}$. An advantage of the approach developed in [6] is that it admits a simple generalization suitable for a description of the (small) quantum cohomology ring of flag varieties, as well as the (quantum) Schubert polynomials. The constructions of [6] were generalized to other finite root systems by the authors in [9. A principal constituent of the above constructions is the Dunkl elements. The basic properties of the Dunkl elements are as follows:

1) they are pairwise commuting;

2) in the so-called Calogero-Moser representation 6, 9, they correspond to the truncated (i.e., without differential part) rational Dunkl operators [4];

3 ) in the crystallographic case they correspond - after applying the so-called Bruhat representation [6, 9] — to the Monk formula in the cohomology ring of the flag variety in question;

4) in the crystallographic case, subtraction-free expressions of Schubert polynomials calculated at the Dunkl elements in the algebra $\widetilde{\mathcal{B E}}(\Delta)$ should provide a combinatorial rule for describing the Schubert basis structural constants, i.e., the intersection numbers of Schubert classes.

In the case of classical root systems $\Delta$, the first author [7] defined a certain extension $\widetilde{\mathcal{B E}}(\Delta)$ of the algebra $\mathcal{B E}(\Delta)$, together with a pairwise commuting family of elements, called the Dunkl elements, which after applying the Calogero-Moser representation

2010 Mathematics Subject Classification. Primary 05E15, 14M15.

Key words and phrases. Root system of type $A$, equivariant Pieri rule, Nichols-Woronowicz algebra. 
coincide exactly with the rational Dunkl operators. We recall that the Calogero-Moser representation of the algebra $\widetilde{\mathcal{B E}}(\Delta)$ is a representation given by the divided difference operators $\partial_{\alpha}:=\left(1-s_{\alpha}\right) / \alpha$ acting on the symmetric algebra $S(V)$, where $V$ is a reflection representation of the Weyl group corresponding to the root system $\Delta$, and $s_{\alpha}$ is a reflection with respect to the root $\alpha \in \Delta$.

One of our main objectives in this paper is to study the commutative subalgebra generated by the Dunkl elements in the extended algebra $\widetilde{\mathcal{B E}}(\Delta)$ in the case of type $A$ root systems. Postnikov [13] proved a Pieri-type formula for the elementary symmetric polynomials evaluated at the Dunkl elements, which had been originally conjectured in [6]. In Theorem 2.1 we show an analog of the Pieri-type formula in the extended algebra $\widetilde{\mathcal{B E}}(\Delta)$ of type $A$. As a consequence, it is shown that the commutative subalgebra generated by the Dunkl elements is isomorphic to the equivariant cohomology ring $H_{T}^{*}\left(F l_{n}\right)$, where $F l_{n}$ is the flag variety parametrizing the full flags in the vector space $\mathbb{C}^{n}$, and $T=\left(\mathbb{C}^{\times}\right)^{n}$ is the torus acting on $\mathbb{C}^{n}$ diagonally.

In $\S 3$ we construct the Bruhat representation of the algebra $\mathcal{E}_{n}\langle R\rangle[t]$ and study some properties of the former. The existence of Bruhat's representation of the algebra $\mathcal{E}_{n}\langle R\rangle[t]$ plays a crucial role in applications to the equivariant Schubert calculus and constitutes an important step in the construction of the model of $H_{T}^{*}\left(F l_{n}\right)$. Our formula in Theorem 2.1 describes the Pieri formula for the equivariant cohomology ring $H_{T}^{*}\left(F l_{n}\right)$ via the Bruhat representation. The Pieri formula for double Schubert polynomials was studied in [15, Chapter 5]. A similar rule in the cohomology ring $H_{T}^{*}\left(F l_{n}\right)$ was stated and proved in [14. We shall show how to compute the multiplication by the special Schubert classes via the Bruhat representation in Example 3.1, where an example is also given to see that the result of the computation based on our formula coincides with that in [14.

In $\S 4$ we introduce and study the "quantum" deformation $\mathcal{E}_{n}^{\mathbf{q}}$ and $\mathcal{E}_{n}^{\mathbf{q}}\langle R\rangle[t]$ of the algebras $\mathcal{E}_{n}$ and $\mathcal{E}_{n}\langle R\rangle[t]$. It is remarkable that the description of the Pieri-type formula based on the quadratic algebra $\mathcal{E}_{n}^{\mathbf{q}}$ gives the multiplication rule in the quantum cohomology ring $Q H^{*}\left(F l_{n}\right)$ under a natural quantum deformation of the algebra $\mathcal{E}_{n}$ presented in Definition 4.1. As shown in $\S 4$, our formula also covers a similar formula in the equivariant quantum cohomology ring $Q H_{T}^{*}\left(F l_{n}\right)$.

Another objective in our paper is to construct a certain extension of the NicholsWoronowicz model for the coinvariant algebra of a finite Coxeter group $W$. It is conjectured that the Fomin-Kirillov quadratic algebra $\mathcal{E}_{n}$ is isomorphic to the NicholsWoronowicz algebra associated with a certain kind of Yetter-Drinfeld module defined by the data of the root system of type $A_{n-1}$. More generally, the algebra $\mathcal{B E}(\Delta)$ is a lift of the Nichols-Woronowicz algebra for the root system $\Delta$. Recall that the Nichols-Woronowicz algebra model for the cohomology ring of flag varieties was invented by Y. Bazlov [2]. In $\S 4$ we introduce a certain extension $\widetilde{\mathcal{B}}_{W}$ of the Nichols-Woronowicz algebra $\mathcal{B}_{W}$ and construct a commutative subalgebra in the extended Nichols-Woronowicz algebra. Our second main result states that, for crystallographic root systems and $t=0$, the commutative subalgebra of $\widetilde{\mathcal{B}}_{W}$ in question is isomorphic to the $T$-equivariant cohomology ring of the corresponding flag variety.

\section{§2. Extension of the QUADRATIC ALGEBRA}

Definition 2.1. The algebra $\mathcal{E}_{n}$ is an associative algebra generated by the symbols $[i, j]$, $1 \leq i, j \leq n, i \neq j$, subject to the relations

(0) $[i, j]=-[j, i]$,

(1) $[i, j]^{2}=0$,

(2) $[i, j][k, l]=[k, l][i, j]$ if $\{i, j\} \cap\{k, l\}=\varnothing$,

(3) $[i, j][j, k]+[j, k][k, i]+[k, i][i, j]=0$. 
We consider the extension $\mathcal{E}_{n}\langle R\rangle[t]$ of the quadratic algebra $\mathcal{E}_{n}$ by the polynomial ring $R[t]=\mathbb{Z}\left[x_{1}, \ldots, x_{n}\right][t]$ defined by the following commutation relations:

(A) $[i, j] x_{k}=x_{k}[i, j]$, for $k \neq i, j$,

(B) $[i, j] x_{i}=x_{j}[i, j]+t,[i, j] x_{j}=x_{i}[i, j]-t$, for $i<j$,

(C) $[i, j] t=t[i, j]$.

Note that the $\mathbf{S}_{n}$-invariant subalgebra $R^{\mathbf{S}_{n}}[t]$ of $R[t]$ is contained in the center of the algebra $\mathcal{E}_{n}\langle R\rangle[t]$. Let $e_{k}\left(x_{1}, \ldots, x_{n}\right), 1 \leq k \leq n$, stand for the elementary symmetric polynomial of degree $k$ in the variables $x_{1}, \ldots, x_{n}$. By definition, we put $e_{0}\left(x_{1}, \ldots, x_{n}\right)=1$, and $e_{k}\left(x_{1}, \ldots, x_{n}\right)=0$ if $k<0$.

Definition 2.2. (1) We define the $R[t]$-algebra $\widetilde{\mathcal{E}}_{n}[t]$ by

$$
\widetilde{\mathcal{E}}_{n}[t]=\mathcal{E}_{n}\langle R\rangle[t] \otimes_{R^{\mathbf{s}_{n}}} R
$$

More explicitly, $\widetilde{\mathcal{E}}_{n}[t]$ is an algebra over the polynomial ring $\mathbb{Z}\left[y_{1}, \ldots, y_{n}\right]$ generated by the symbols $[i, j], 1 \leq i, j \leq n, i \neq j$, and $x_{1}, \ldots, x_{n}, t$ satisfying the relations in the definition of the algebra $\mathcal{E}_{n}\langle R\rangle[t]$, together with the identification $e_{i}\left(x_{1}, \ldots, x_{n}\right)=e_{i}\left(y_{1}, \ldots, y_{n}\right)$ for $i=1, \ldots, n$. Let $\widetilde{\mathcal{E}}_{n, t_{0}}$ denote the specialization of $\widetilde{\mathcal{E}}_{n}[t]$ at $t=t_{0}$.

(2) The Dunkl elements $\theta_{i} \in \widetilde{\mathcal{E}}_{n}[t], i=1, \ldots, n$, are defined by the formula

$$
\theta_{i}=\theta_{i}^{(n)}=x_{i}+\sum_{j \neq i}[i, j]
$$

Remark 2.1. Note that the $x_{i}$ 's do not commute with the Dunkl elements, but only symmetric polynomials in the $x_{i}$ 's do. Moreover, we need the $R$-algebra structure of $\widetilde{\mathcal{E}}_{n}[t]$ to construct the model of the $T$-equivariant cohomology ring $H_{T}^{*}\left(F l_{n}\right)$, which is an algebra over $H_{T}^{*}$ (pt.) $\cong R$. For this reason, we need the second copy of $R=\mathbb{Z}\left[y_{1}, \ldots, y_{n}\right]$, where the $y_{i}$ 's are assumed to belong to the center of the algebra $\widetilde{\mathcal{E}}_{n}[t]$, and $f\left(x_{1}, \ldots, x_{n}\right)=$ $f\left(y_{1}, \ldots, y_{n}\right)$ for any symmetric polynomial $f$.

Lemma 2.1. The Dunkl elements commute with each other.

Proof. This follows from the fact that

$$
\left(x_{i}+x_{j}\right)[i, j]=[i, j]\left(x_{i}+x_{j}\right) .
$$

Theorem 2.1 (Pieri formula in the algebra $\mathcal{E}_{n}\langle R\rangle[t]$ ). For $k \leq m \leq n$, we have

$$
e_{k}\left(\theta_{1}^{(n)}, \ldots, \theta_{m}^{(n)}\right)=\sum_{r \geq 0}(-t)^{r} N(m-k, 2 r)\left\{\sum_{S, I=\left\{i_{a}\right\},\left(j_{a}\right)} X_{S} \cdot\left[i_{1}, j_{1}\right] \cdots\left[i_{|I|}, j_{|I|}\right]\right\}
$$

where

$$
N(a, 2 b)=(2 b-1) ! !\left(\begin{array}{c}
a+2 b \\
2 b
\end{array}\right)
$$

$X_{S}:=\prod_{s \in S} x_{s}$, and the second summation runs over the triples

$$
\left(S, I=\left\{i_{1}, \ldots, i_{|I|}\right\},\left(j_{a}\right)_{a=1}^{|I|}\right)
$$

such that $S \subset\{1, \ldots, m\} ; I$ is a subset of $\{1, \ldots, m\} \backslash S ;|I|+|S|+2 r=k ; 1 \leq i_{a} \leq$ $m<j_{a} \leq n ;$ and $j_{1} \leq \cdots \leq j_{|I|}$.

Proof. Let $\mathcal{A}$ be a subset of $\{1, \ldots, n\}$; we put $m:=|\mathcal{A}|, d:=n-m$, and $\{1, \ldots, n\} \backslash \mathcal{A}=$ $\left\{j_{1}<\cdots<j_{d}\right\}$. Denote by $E_{k}(\mathcal{A})$ the right-hand side of the formula, i.e.,

$$
E_{k}(\mathcal{A}):=\sum_{r \geq 0}(-t)^{r} N(m-k, 2 r) \sum_{S \subset \mathcal{A}} X_{S} \sum_{(*)}\left[s_{1}, t_{1}\right] \cdots\left[s_{k-2 r-|S|}, t_{k-2 r-|S|}\right],
$$

where $(*)$ stands for the following conditions: the elements $s_{1}, \ldots, s_{k-2 r-|S|} \in \mathcal{A} \backslash S$ are 
distinct, $t_{1}, \ldots, t_{k-2 r-|S|} \in\{1, \ldots, n\} \backslash \mathcal{A}$, and $t_{1} \leq \cdots \leq t_{k-2 r-|S|}$. It will suffice to prove the recursive formula

$$
E_{k}\left(\mathcal{A} \cup\left\{j=j_{1}\right\}\right)=E_{k}(\mathcal{A})+E_{k-1}(\mathcal{A})\left(x_{j}+\sum_{s \neq j}[j, s]\right) .
$$

For a subset $I=\left\{i_{1}, \ldots, i_{l}\right\} \subset\{1, \ldots, n\}$ and $p \notin I$, we use the symbol

$$
\langle\langle I \mid p\rangle\rangle=\sum_{w \in \mathbf{S}_{l}}\left[i_{w(1)}, p\right] \cdots\left[i_{w(l)}, p\right]
$$

as defined in 13. We also use the symbol $I_{1} \cdots I_{d} \subset_{m} I$, which means that $I_{1}, \ldots, I_{d} \subset I$ are disjoint and $\# I_{1}+\cdots+\# I_{d}=m$. We have the following decompositions:

$$
\begin{aligned}
& E_{k}(\mathcal{A}) \\
& =\sum_{r \geq 0}(-t)^{r} N(m-k, 2 r) \sum_{S \subset \mathcal{A}} X_{S} \sum_{I_{1} \cdots I_{d} \subset k-2 r-|S| \mathcal{A} \backslash S}\left\langle\left\langle I_{1} \mid j_{1}\right\rangle\right\rangle \cdots\left\langle\left\langle I_{d} \mid j_{d}\right\rangle\right\rangle \\
& =\sum_{r \geq 0}(-t)^{r} N(m-k, 2 r)\left(A_{1}^{r}+A_{2}^{r}\right), \\
& E_{k}(\mathcal{A} \cup\{j\}) \\
& =\sum_{r \geq 0}(-t)^{r} N(m-k+1,2 r) \sum_{S \subset \mathcal{A}} X_{S} \sum_{I_{2} \cdots I_{d} \subset_{k-2 r-|S|} \mathcal{A} \cup\{j\} \backslash S}\left\langle\left\langle I_{2} \mid j_{2}\right\rangle\right\rangle \cdots\left\langle\left\langle I_{d} \mid j_{d}\right\rangle\right\rangle \\
& =\sum_{r \geq 0}(-t)^{r} N(m-k+1,2 r)\left(B_{1}^{r}+B_{2}^{r}+B_{3}^{r}\right), \\
& E_{k-1}(\mathcal{A}) \sum_{s \neq j}[j, s] \\
& =\sum_{r \geq 0}(-t)^{r} N(m-k+1,2 r) \sum_{S \subset \mathcal{A}} X_{S} \sum_{I_{1} \cdots I_{d} \subset_{k-1-2 r-|S|} \mathcal{A} \backslash S}\left\langle\left\langle I_{1} \mid j_{1}\right\rangle\right\rangle \cdots\left\langle\left\langle I_{d} \mid j_{d}\right\rangle\right\rangle \sum_{s \neq j}[j, s] \\
& =\sum_{r \geq 0}(-t)^{r} N(m-k+1,2 r)\left(C_{1}^{r}+C_{2}^{r}+C_{3}^{r}+C_{4}^{r}\right), \\
& E_{k-1}(\mathcal{A}) x_{j} \\
& =\sum_{r \geq 0}(-t)^{r} N(m-k+1,2 r) \sum_{S \subset \mathcal{A}} X_{S} \sum_{I_{1} \cdots I_{d} \subset k-1-2 r-|S| \mathcal{A} \backslash S}\left\langle\left\langle I_{1} \mid j_{1}\right\rangle\right\rangle \cdots\left\langle\left\langle I_{d} \mid j_{d}\right\rangle\right\rangle x_{j} \\
& =\sum_{r \geq 0}(-t)^{r} N(m-k+1,2 r)\left(D_{1}^{r}+D_{2}^{r}\right) \text {, }
\end{aligned}
$$

where $A_{i}^{r}, B_{i}^{r}, C_{i}^{r}, D_{i}^{r}$ are defined as follows.

- $A_{1}^{r}$ is the sum of terms with $I_{1}=\varnothing ; A_{2}^{r}$ is the sum of terms with $I_{1} \neq \varnothing$.

- $B_{1}^{r}$ is the sum of terms with $j \notin S \cup I_{2} \cup \cdots \cup I_{d} ; B_{2}^{r}$ is the sum of terms with $j \in I_{2} \cup \cdots \cup I_{d} ; B_{3}^{r}$ is the sum of terms with $j \in S$.

- $C_{1}^{r}$ is the sum of terms with $s \in \mathcal{A} \backslash\left(S \cup I_{1} \cup \cdots \cup I_{d}\right) ; C_{2}^{r}$ is the sum of terms with $s \in I_{2} \cup \cdots \cup I_{d} \cup \mathcal{A}^{c} ; C_{3}^{r}$ is the sum of terms with $s \in S ; C_{4}^{r}$ is the sum of terms with $s \in I_{1}$.

- $D_{1}^{r}$ is the sum of terms with $I_{1}=\varnothing ; D_{2}^{r}$ is the sum of terms with $I_{1} \neq \varnothing$.

The same arguments as in [13] show that $A_{1}^{r}=B_{1}^{r}, A_{2}^{r}+C_{1}^{r}=0, B_{2}^{r}=C_{2}^{r}$, and 
$C_{4}^{r}=0$. It is also easy to check that $B_{3}^{r}=D_{1}^{r}$. Now we have

$$
\begin{aligned}
E_{k}(\mathcal{A})+ & E_{k-1}(\mathcal{A})\left(x_{j}+\sum_{s \neq j}[j, s]\right)-E_{k}(\mathcal{A} \cup\{j\}) \\
= & \sum_{r \geq 0}(-t)^{r}\left(N(m-k, 2 r)\left(A_{1}^{r}+A_{2}^{r}\right)-N(m-k+1,2 r)\left(B_{1}^{r}-C_{1}^{r}-C_{3}^{r}-D_{2}^{r}\right)\right) \\
= & \sum_{r \geq 1}(-t)^{r}(N(m-k, 2 r)-N(m-k+1,2 r))\left(A_{1}^{r}+A_{2}^{r}\right) \\
& +\sum_{r \geq 0}(-t)^{r} N(m-k+1,2 r)\left(C_{3}^{r}+D_{2}^{r}\right) .
\end{aligned}
$$

From the commutation relation $[i, j] x_{j}=x_{i}[i, j]-t$, we have

$$
\begin{aligned}
& D_{2}^{r}=\sum_{S \subset \mathcal{A}} X_{S} \sum_{\substack{I_{1} \cdots I_{d} \subset k-1-2 r-|S| \\
I_{1}=\left\{a_{1}, \ldots, a_{\left|I_{1}\right|}\right\}}} \\
& \times \sum_{w \in \mathbf{S}_{\left|I_{1}\right|}} x_{a_{w\left(\left|I_{1}\right|\right)}}\left[a_{w(1)}, j\right] \cdots\left[a_{w\left(\left|I_{1}\right|\right)}, j\right]\left\langle\left\langle I_{2} \mid j_{2}\right\rangle\right\rangle \cdots\left\langle\left\langle I_{d} \mid j_{d}\right\rangle\right\rangle \\
& -t \sum_{S \subset \mathcal{A}} X_{S} \sum_{\substack{I_{1} \cdots I_{d} \subset k-1-2 r-|S| \\
I_{1}=\left\{a_{1}, \ldots, a_{\left|I_{1}\right|}\right\}}} \sum_{\substack{w \in \mathbf{S}_{\left|I_{1}\right|}\\
}}\left[a_{w(1)}, j\right] \cdots\left[a_{w\left(\left|I_{1}\right|-1\right)}, j\right]\left\langle\left\langle I_{2} \mid j_{2}\right\rangle\right\rangle \cdots\left\langle\left\langle I_{d} \mid j_{d}\right\rangle\right\rangle \\
& =\sum_{S \subset \mathcal{A}} \sum_{s \notin S} X_{S \cup\{s\}} \sum_{I_{1} \cdots I_{d} \subset_{k-1-2 r-(|S|+1)} \mathcal{A} \backslash S \cup\{s\}}\left\langle\left\langle I_{1} \mid j_{1}\right\rangle\right\rangle[s, j]\left\langle\left\langle I_{2} \mid j_{2}\right\rangle\right\rangle \cdots\left\langle\left\langle I_{d} \mid j_{d}\right\rangle\right\rangle \\
& -(m-k+2 r+2) t \sum_{S \subset \mathcal{A}} X_{S} \sum_{I_{1} \cdots I_{d} \subset{ }_{k-2-2 r-|S| \mathcal{A} \backslash S}}\left\langle\left\langle I_{1} \mid j_{1}\right\rangle\right\rangle \cdots\left\langle\left\langle I_{d} \mid j_{d}\right\rangle\right\rangle \\
& =-C_{3}^{r}+(-t)(m-k+2 r+2)\left(A_{1}^{r+1}+A_{2}^{r+1}\right) .
\end{aligned}
$$

Hence,

$$
\begin{aligned}
(-t)^{r+1} & \left(N(m-k, 2(r+1))-N(m-k+1,2(r+1))\left(A_{1}^{r+1}+A_{2}^{r+1}\right)\right. \\
& =-(-t)^{r+1}(2 r+1) ! ! \frac{(m-k+2 r+2) !}{(2 r+1) !(m-k+1) !}\left(A_{1}^{r+1}+A_{2}^{r+1}\right) \\
& =-(-t)^{r}(2 r-1) ! ! \frac{(m-k+2 r+1) !}{(2 r) !(m-k+1) !} \cdot(-t)(m-k+2 r+2)\left(A_{1}^{r+1}+A_{2}^{r+1}\right) \\
& =-(-t)^{r} N(m-k+1,2 r)\left(C_{3}^{r}+D_{2}^{r}\right) .
\end{aligned}
$$

This implies the desired result.

Example 2.1. Let us check the coefficients of $t$ and $t^{2}$ in the expression of $\theta_{1} \theta_{2} \theta_{3} \theta_{4} \in$ $\mathcal{E}_{5}\langle R\rangle[t]$ by direct computation. It is easily seen that

$$
\begin{aligned}
\theta_{1} \theta_{2} \theta_{3}= & -t\left(x_{1}+x_{2}+x_{3}+[14]+[15]+[24]+[25]+[34]+[35]\right) \\
& +x_{1} x_{2} x_{3}+x_{1} x_{2}([34]+[35])+x_{1} x_{3}([24]+[25])+x_{2} x_{3}([14]+[15]) \\
& +x_{1}([24][34]+[24][35]+[25][35]+[34][24]+[34][25]+[35][25]) \\
& +x_{2}([14][34]+[14][35]+[15][35]+[34][14]+[34][15]+[35][15]) \\
& +x_{3}([14][24]+[14][25]+[15][25]+[24][14]+[24][15]+[25][15]) \\
& +\sum_{\substack{\left\{i_{1}, i_{2}, i_{3}\right\}=\{1,2,3\} \\
4 \leq j_{1} \leq j_{2} \leq j_{3} \leq 5}}\left[i_{1} j_{1}\right]\left[i_{2} j_{2}\right]\left[i_{3} j_{3}\right] .
\end{aligned}
$$


We multiply this expression by $\theta_{4}$ from the right. The contributions to the coefficients of $t$ and $t^{2}$ may come only from the following terms:

- $-t\left(x_{1}+x_{2}+x_{3}+[14]+[15]+[24]+[25]+[34]+[35]\right) \theta_{4}$

$$
\begin{aligned}
=3 t^{2}-t & \left(x_{1} x_{4}+x_{2} x_{4}+x_{3} x_{4}+x_{1}([42]+[43]+[45])\right. \\
& +x_{2}([41]+[43]+[45])+x_{3}([41]+[42]+[45])+x_{4}([15]+[25]+[35]) \\
& +[15][41]+[24][41]+[25][41]+[34][41]+[35][41] \\
& +[14][42]+[15][42]+[25][42]+[34][42]+[35][42] \\
& +[14][43]+[15][43]+[24][43]+[25][43]+[35][43] \\
& +[14][45]+[15][45]+[24][45]+[25][45]+[34][45]+[35][45])
\end{aligned}
$$

- $\quad x_{1}([24][34]+[24][35]+[25][35]+[34][24]+[34][25]+[35][25]) x_{4}$

$$
=-t x_{1}([24]+[34]+[25]+[35])+\cdots,
$$

- $\quad x_{2}([14][34]+[14][35]+[15][35]+[34][14]+[34][15]+[35][15]) x_{4}$

$$
=-t x_{2}([14]+[34]+[15]+[35])+\cdots,
$$

- $x_{3}([24][14]+[24][15]+[25][15]+[14][24]+[14][25]+[15][25]) x_{4}$

$$
=-t x_{3}([24]+[14]+[25]+[15])+\cdots,
$$

- $x_{1} x_{2}([34]+[35]) x_{4}=-t x_{1} x_{2}+x_{1} x_{2}\left(x_{3}[34]+x_{4}[35]\right)$,

- $x_{2} x_{3}([14]+[15]) x_{4}=-t x_{2} x_{3}+x_{2} x_{3}\left(x_{1}[14]+x_{4}[15]\right)$,

- $x_{1} x_{3}([24]+[25]) x_{4}=-t x_{1} x_{3}+x_{1} x_{3}\left(x_{2}[24]+x_{4}[25]\right)$,

- $\sum_{\left\{i_{1}, i_{2}, i_{3}\right\}=\{1,2,3\}}\left[i_{1} j_{1}\right]\left[i_{2} j_{2}\right]\left[i_{3} j_{3}\right] x_{4}$

$$
\begin{aligned}
& 4 \leq j_{1} \leq j_{2} \leq j_{3} \leq 5 \\
& =-t([14][24]+[14][35]+[25][35]+[24][14]+[24][35]+[15][35] \\
& +[34][14]+[34][25]+[15][25]+[34][24]+[34][15]+[25][15] \\
& +[14][34]+[14][25]+[35][25]+[24][34]+[24][15]+[35][15])+\cdots \text {. }
\end{aligned}
$$

By using the relations $[15][41]+[14][45]=[45][15],[25][42]+[24][45]=[45][25],[35][43]+$ $[34][45]=[45][35]$, we obtain finally

$$
\begin{aligned}
\theta_{1} \theta_{2} \theta_{3} \theta_{4}=3 t^{2}-t & \left(x_{1} x_{2}+x_{1} x_{3}+x_{1} x_{4}+x_{2} x_{3}+x_{2} x_{4}+x_{3} x_{4}\right. \\
& +x_{1}([25]+[35]+[45])+x_{2}([15]+[35]+[45]) \\
& +x_{3}([15]+[25]+[45])+x_{4}([15]+[25]+[35]) \\
& +[15][25]+[15][35]+[15][45]+[25][35]+[25][45]+[35][45] \\
& +[25][15]+[35][15]+[45][15]+[35][25]+[45][25]+[45][35])+\cdots
\end{aligned}
$$

It is easy to show that the formula for $\theta_{1} \theta_{2} \theta_{3} \theta_{4}$ stated in Theorem 2.1 produces the same expression.

The following is a special case of the formula in Theorem 2.1 for $m=n$.

Corollary 2.1. In the algebra $\widetilde{\mathcal{E}}_{n}[t]$, we have the relations

$$
\begin{aligned}
& e_{k}\left(\theta_{1}^{(n)}, \ldots, \theta_{n}^{(n)}\right)=e_{k}\left(y_{1}, \ldots, y_{n}\right)+\sum_{r \geq 1}(-t)^{r}(2 r-1) ! !\left(\begin{array}{c}
n-k+2 r \\
2 r
\end{array}\right) e_{k-2 r}\left(y_{1}, \ldots, y_{n}\right), \\
& 1 \leq k \leq n .
\end{aligned}
$$


In $\S 3$ it will be shown that the above relations describe a complete set of relations among the Dunkl elements in $\widetilde{\mathcal{E}}_{n}[t]$.

\section{§3. Bruhat REPRESEntation}

We recall the definition of the Bruhat representation of the algebra $\mathcal{E}_{n}$ on the group ring of the symmetric group $\mathbb{Z}\left\langle\mathbf{S}_{n}\right\rangle=\bigoplus_{w \in \mathbf{S}_{n}} \mathbb{Z} \cdot \underline{w}$. The operator $\sigma_{i j}, i<j$, is defined as follows:

$$
\sigma_{i j}(\underline{w})= \begin{cases}\frac{w t_{i j}}{0} & \text { if } l\left(w t_{i j}\right)=l(w)+1, \\ 0 & \text { otherwise }\end{cases}
$$

where $t_{i j} \in \mathbf{S}_{n}$ is the transposition of $i$ and $j$. Then the Bruhat representation of $\mathcal{E}_{n}$ is defined by $[i, j] . \underline{w}:=\sigma_{i j}(\underline{w})$.

Now we extend the Bruhat representation to that of the algebra $\mathcal{E}_{n}\langle R\rangle[t]$ defined on

$$
R[t]\left\langle\mathbf{S}_{n}\right\rangle=\bigoplus_{w \in \mathbf{S}_{n}} \mathbb{Z}\left[y_{1}, \ldots, y_{n}\right][t] \cdot \underline{w} .
$$

We define the divided difference operator $\partial_{i j}$ on $\mathbb{Z}\left[y_{1}, \ldots, y_{n}\right][t]$ as the $\mathbb{Z}[t]$-linear operator given by $\partial_{i j}:=\left(1-t_{i j}\right) /\left(y_{i}-y_{j}\right)$. For $f(y) \in \mathbb{Z}\left[y_{1}, \ldots, y_{n}\right][t]$ and $w \in \mathbf{S}_{n}$, we define the $\mathbb{Z}[t]$-linear operators $\widetilde{\sigma}_{i j}, i<j$, and $\xi_{k}$ as follows:

$$
\begin{aligned}
\widetilde{\sigma}_{i j}(f(y) \underline{w}) & = \begin{cases}t\left(\partial_{w(i) w(j)} f(y)\right) \underline{w}+f(y) \underline{w t_{i j}} & \text { if } l\left(w t_{i j}\right)=l(w)+1, \\
t\left(\partial_{w(i) w(j)} f(y)\right) \underline{w} & \text { otherwise, }\end{cases} \\
\xi_{k}(f(y) \underline{w}) & =\left(y_{w(k)} f(y)\right) \underline{w} .
\end{aligned}
$$

Proposition 3.1. The algebra $\mathcal{E}_{n}\langle R\rangle[t]$ acts $\mathbb{Z}[t]$-linearly on $\mathbb{Z}[y][t]\left\langle\mathbf{S}_{n}\right\rangle$ via $[i j] \mapsto \widetilde{\sigma}_{i j}$ and $x_{k} \mapsto \xi_{k}$.

Proof. We check compatibility with the defining relations of the algebra $\widetilde{\mathcal{E}}_{n}[t]$. We show this only for relations (1), (3), and (B). The rest is easy to check.

We start with relation (1). We have

$$
\begin{aligned}
\tilde{\sigma}_{i j}^{2}(f(y) \underline{w})= & \widetilde{\sigma}_{i j}\left(t\left(\partial_{w(i) w(j)} f(y)\right) \underline{w}+f(y) \sigma_{i j}(\underline{w})\right) \\
= & t^{2}\left(\partial_{w(i) w(j)}^{2} f(y)\right) \underline{w} \\
& +t\left(\partial_{w(i) w(j)} f(y)\right) \sigma_{i j}(\underline{w}) \\
& +t\left(\partial_{w(j) w(i)} f(y)\right) \sigma_{i j}(\underline{w})+f(y) \sigma_{i j}^{2}(\underline{w}) .
\end{aligned}
$$

Since $\partial_{w(i) w(j)}^{2}=0, \sigma_{i j}^{2}=0$ and $\partial_{w(i) w(j)}=-\partial_{w(j) w(i)}$, we get $\widetilde{\sigma}_{i j}^{2}=0$.

For relation (3), we have

$$
\begin{aligned}
\tilde{\sigma}_{i j} \widetilde{\sigma}_{j k}(f(y) \underline{w})= & \widetilde{\sigma}_{i j}\left(t\left(\partial_{w(j) w(k)} f(y)\right) \underline{w}+f(y) \sigma_{j k}(\underline{w})\right) \\
= & t^{2}\left(\partial_{w(i) w(j)} \partial_{w(j) w(k)} f(y)\right) \underline{w} \\
& +t\left(\partial_{w(j) w(k)} f(y)\right) \sigma_{i j}(\underline{w}) \\
& +t\left(\partial_{w(i) w(k)} f(y)\right) \sigma_{j k}(\underline{w})+f(y) \sigma_{i j} \sigma_{j k}(\underline{w}) .
\end{aligned}
$$

We also obtain $\widetilde{\sigma}_{j k} \widetilde{\sigma}_{k i}(f(y) \underline{w})$ and $\widetilde{\sigma}_{k i} \widetilde{\sigma}_{i j}(f(y) \underline{w})$ by the cyclic permutation of $i, j, k$. The 3 -term relations

$$
\partial_{w(i) w(j)} \partial_{w(j) w(k)}+\partial_{w(j) w(k)} \partial_{w(k) w(i)}+\partial_{w(k) w(i)} \partial_{w(i) w(j)}=0
$$

and

$$
\sigma_{i j} \sigma_{j k}+\sigma_{j k} \sigma_{k i}+\sigma_{k i} \sigma_{i j}=0
$$

show the desired identity

$$
\widetilde{\sigma}_{i j} \widetilde{\sigma}_{j k}+\widetilde{\sigma}_{j k} \widetilde{\sigma}_{k i}+\widetilde{\sigma}_{k i} \widetilde{\sigma}_{i j}=0 .
$$


Finally, we check relation (B). We have

$$
\begin{aligned}
\widetilde{\sigma}_{i j} \xi_{i}(f(y) \underline{w}) & =\widetilde{\sigma}_{i j}\left(y_{w(i)} f(y) \underline{w}\right) \\
& =t \partial_{w(i) w(j)}\left(y_{w(i)} f(y)\right) \underline{w}+\left(y_{w(i)} f(y)\right) \sigma_{i j}(\underline{w}) \\
& =t(f(y) \underline{w})+t\left(y_{w(j)} \partial_{w(i) w(j)} f(y)\right) \underline{w}+y_{w t_{i j}(j)} \sigma_{i j}(\underline{w}) \\
& =\xi_{j} \widetilde{\sigma}_{i j}(f(y) \underline{w})+t(f(y) \underline{w}) .
\end{aligned}
$$

This completes the proof of the fact that the Bruhat representation is well defined.

Let $A H_{n}^{0}$ denote the subalgebra in $\mathcal{E}_{n}\langle R\rangle[t]$ generated by the elements $t, h_{1}:=[1,2]$, $h_{2}:=[2,3], \ldots, h_{n-1}:=[n-1, n]$, and $x_{1}, \ldots, x_{n}$.

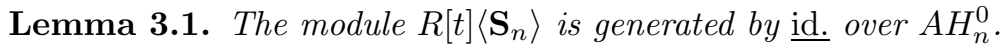

Proof. Consider a reduced decomposition $w=s_{i_{1}} \cdots s_{i_{l}}, s_{i}=(i, i+1)$, of a permutation $w \in \mathbf{S}_{n}$; then

$$
h_{i_{l}} \cdots h_{i_{1}}(\underline{\mathrm{id}} .)=\underline{w} .
$$

Therefore, $R[t]\left\langle\mathbf{S}_{n}\right\rangle=A H_{n}^{0} \cdot \underline{\mathrm{id}}$.

Theorem 3.1. The subalgebra $A H_{n}^{0}$ of $\mathcal{E}_{n}\langle R\rangle[t]$ is isomorphic to the nil degenerate affine Hecke algebra $\mathcal{A} H_{n}^{0}$ of type $A_{n-1}^{(1)}$, i.e., the $\mathbb{Z}[t]$-algebra given by two sets of generators $g_{1}, \ldots, g_{n-1}$ and $x_{1}, \ldots, x_{n}$ subject to the following set of defining relations:

$$
\begin{aligned}
& g_{i}^{2}=0 ; \quad g_{i} g_{j}=g_{j} g_{i} \quad \text { if } \quad|i-j|>1 ; \quad g_{i} g_{j} g_{i}=g_{j} g_{i} g_{j} \quad \text { if }|i-j|=1 ; \\
& x_{i} x_{j}=x_{j} x_{i}, \quad x_{k} g_{i}=g_{i} x_{k} \quad \text { if } k \neq i, i+1 ; \quad g_{i} x_{i}-x_{i+1} g_{i}=t .
\end{aligned}
$$

Proof. First, it is easy to check that the elements $t, h_{1}, \ldots, h_{n-1}, x_{1}, \ldots, x_{n}$ do satisfy the relations listed above. Hence, we have a surjective homomorphism $\rho$ from the nil degenerate affine Hecke algebra $\mathcal{A} H_{n}^{0}$ to $A H_{n}^{0}$, given by $\rho\left(g_{i}\right)=h_{i}$. Now we are going to construct a basis in the algebra $\mathcal{A} H_{n}^{0}$. Let $w \in \mathbf{S}_{n}$ be a permutation and $w=s_{i_{1}} \cdots s_{i_{k}}$ a reduced decomposition of it. Since the elements $g_{1}, \ldots, g_{n-1}$ satisfy the Coxeter relations, the element $g_{w}:=g_{i_{1}} \cdots g_{i_{k}}$ is well defined. On the other hand, using relations among the elements $x_{1}, \ldots, x_{n}$ and $g_{1}, \ldots, g_{n-1}$ in the algebra $\mathcal{A} H_{n}^{0}$, we can write any element of $\mathcal{A} H_{n}^{0}$ as a linear combination of elements $t^{a} x^{m} g_{w}$, where $w \in \mathbf{S}_{n}, m \in \mathbb{Z}_{\geq 0}^{n}$, and $a \in \mathbb{Z}_{\geq 0}$. Lemma 3.1 implies that $R[t]\left\langle\mathbf{S}_{n}\right\rangle=\rho\left(\mathcal{A} H_{n}^{0}\right) \cdot$ id., so that the elements $\left\{t^{a} x^{m} g_{w} \mid a \in\right.$ $\left.\mathbb{Z}_{\geq 0}, w \in \mathbf{S}_{n}, m \in \mathbb{Z}_{\geq 0}^{n}\right\}$ must be linearly independent over $\mathbf{Z}$. This yields the injectivity of $\rho$, and we conclude that the homomorphism $\rho$ is an isomorphism.

Let us consider the double Schubert polynomials $\mathfrak{S}_{w}(x, y), w \in \mathbf{S}_{n}$, introduced by Lascoux and Schützenberger [10. By definition, the polynomial $\mathfrak{S}_{w_{0}}(x, y)$ for the maximal element $w_{0} \in \mathbf{S}_{n}$ is given by $\mathfrak{S}_{w_{0}}(x, y):=\prod_{i+j<n}\left(x_{i}-y_{j}\right)$. For $w \in \mathbf{S}_{n}$, take a reduced decomposition $w=s_{i_{1}} \cdots s_{i_{l}}$. The divided difference operator $\partial_{w}:=\partial_{i_{1}} \cdots \partial_{i_{l}}$ is well defined, thanks to the Coxeter relations. We define the double Schubert polynomial for $w$ by $\mathfrak{S}_{w}(x, y):=\partial_{w^{-1} w_{0}}^{(x)} \mathfrak{S}_{w_{0}}(x, y)$, where $\partial_{w}^{(x)}$ means a divided difference operator on $x$ variables. The Schubert polynomials are defined to be the specializations $\mathfrak{S}_{w}(x):=\mathfrak{S}_{w}(x, 0)$ of the double Schubert polynomials.

Theorem 3.2. Let $\mathfrak{S}_{w}(x, y)$ be the double Schubert polynomial corresponding to $w \in \mathbf{S}_{n}$. When $t=0$, we have

$$
\mathfrak{S}_{w}(\theta, y)(\underline{\mathrm{id}} .)=\underline{w} .
$$


Proof. This follows from the Monk formula for the double Schubert polynomials (see, e.g., [12, Exercise 2.7.2]) and the relation

$$
\begin{aligned}
\left(\theta_{i}-y_{w(i)}\right)(\underline{w}) & =\xi_{i}(\underline{w})+\sum_{j \neq i} \sigma_{i j}(\underline{w})-y_{w(i)} \underline{w} \\
& =\sum_{\substack{j>i \\
l\left(w t_{i j}\right)=l(w)+1}} \frac{w t_{i j}}{}-\sum_{\substack{j<i \\
l\left(w t_{i j}\right)=l(w)+1}} \underline{w} t_{i j} .
\end{aligned}
$$

Let $w \in \mathbf{S}_{n}$, let $r$ be the maximal descent of $w$, and $s$ the greatest integer such that $w(s)<w(r)$. By applying the Monk formula to the product $x_{r} \mathfrak{S}_{u}(x, y), u=w t_{r s}$, we get the transition formula

$$
\mathfrak{S}_{w}(x, y)=\left(x_{r}-y_{u(r)}\right) \mathfrak{S}_{u}(x, y)+\sum_{v \in S(w, r)} \mathfrak{S}_{v}(x, y),
$$

where $S(w, r)$ is the set of permutations of the form $u t_{j r}=w t_{r s} t_{j r}$ with $j<r$ and of the same length as $w$. Similarly, we have

$$
\underline{w}=\left(\theta_{r}-y_{u(r)}\right) \underline{u}+\sum_{v \in S(w, r)} \underline{v} .
$$

Let $r^{\prime}$ be the maximal descent of $v=w t_{r s} t_{j r} \in S(w, r)$. Since $l(v)=l\left(w t_{r s}\right)+1$, we have $v(r)=w t_{r s}(j)<w t_{r s}(r)=w(s)<w(r)$. Hence, we have either $r^{\prime}<r$ or $\left\{r^{\prime}=r\right.$ and $v(r)<w(r)\}$. Then our assertion is proved by induction on the Bruhat ordering, the maximal descent $r$ and $w(r)$.

Remark 3.1. Only in the case where $t=0$ does the Bruhat representation of the algebra $\mathcal{E}_{n}\langle R\rangle[t]$ admit a $\mathbb{Z}[y][t]$-linear extension up to that of the algebra $\widetilde{\mathcal{E}}_{n, 0}$. In this case, the Dunkl elements commute with multiplication by the $y_{i}$ 's.

Proposition 3.2. The list of relations given in Corollary 2.1 describes a complete set of relations among the Dunkl elements $\theta_{1}^{(n)}, \ldots, \theta_{n}^{(n)}$ in the algebra $\widetilde{\mathcal{E}}_{n}[t]$. In other words, the following surjective homomorphism $\varphi$ between $\mathbb{Z}[t]\left[y_{1}, \ldots, y_{n}\right]$-algebras is an isomorphism:

$$
\begin{array}{cccc}
\varphi: \mathbb{Z}[t]\left[y_{1}, \ldots, y_{n}\right]\left[z_{1}, \ldots, z_{n}\right] / J_{n}^{t} & \rightarrow \mathbb{Z}[t]\left[y_{1}, \ldots, y_{n}\right]\left[\theta_{1}, \ldots, \theta_{n}\right] \subset \widetilde{\mathcal{E}}_{n}[t], \\
z_{i} & \mapsto & \theta_{i},
\end{array}
$$

where the ideal $J_{t}$ is generated by the polynomials

$$
e_{k}\left(z_{1}, \ldots, z_{n}\right)-e_{k}\left(y_{1}, \ldots, y_{n}\right)-\sum_{r \geq 1}(-t)^{r}(2 r-1) ! !\left(\begin{array}{c}
n-k+2 r \\
2 r
\end{array}\right) e_{k-2 r}\left(y_{1}, \ldots, y_{n}\right)
$$

for $k=1, \ldots, n$.

Proof. For the (single) Schubert polynomial $\mathfrak{S}_{w}\left(\theta_{1}, \ldots, \theta_{n}\right) \in \mathcal{E}_{n}\langle R\rangle[t]$ in the Dunkl elements, we have

$$
\left.\mathfrak{S}_{w}\left(\theta_{1}, \ldots, \theta_{n}\right)(\underline{\mathrm{id}} .)\right|_{t=0}=\underline{w}+(\text { linear combination of } \underline{v} \text { with } l(v)<l(w)) .
$$

Consequently,

$$
\begin{aligned}
\mathfrak{S}_{w} & \left(\theta_{1}, \ldots, \theta_{n}\right)(\underline{\mathrm{id} .}) \\
& =\left.\mathfrak{S}_{w}\left(\theta_{1}, \ldots, \theta_{n}\right)(\underline{\mathrm{id}} .)\right|_{t=0}+(\text { linear combination of } \underline{v} \text { with } l(v)<l(w)) \\
& =\underline{w}+(\text { linear combination of } \underline{v} \text { with } l(v)<l(w)) .
\end{aligned}
$$

Hence, the polynomials $\mathfrak{S}_{w}\left(\theta_{1}, \ldots, \theta_{n}\right)$ are linearly independent in $\mathcal{E}_{n}\langle R\rangle[t]$ over $R^{\mathbf{S}_{n}}$. 
Let $R_{\mathbf{S}_{n}}$ be the coinvariant algebra of $\mathbf{S}_{n}$. Since $R=R^{\mathbf{S}_{n}} \otimes R_{\mathbf{S}_{n}}$, the polynomials $\mathfrak{S}_{w}(z), w \in \mathbf{S}_{n}$, form a $\mathbb{Z}[z]^{\mathbf{S}_{n}}$-basis of $\mathbb{Z}[z]$. In particular, any polynomial $f\left(z_{1}, \ldots, z_{n}\right) \in$ $\mathbb{Z}[z]$ can be expressed as

$$
f\left(z_{1}, \ldots, z_{n}\right)=\sum_{w \in \mathbf{S}_{n}} \phi_{w}(z) \mathfrak{S}_{w}(z),
$$

where $\phi_{w}(z) \in \mathbb{Z}[z]^{\mathbf{S}_{n}}=\mathbb{Z}\left[e_{1}(z), \ldots, e_{n}(z)\right]$. It follows that the image of $f\left(z_{1}, \ldots, z_{n}\right)$ in $\mathbb{Z}[t][y]\left[z_{1}, \ldots, z_{n}\right] / J_{n}^{t}$ is a linear combination of $\mathfrak{S}_{w}(z)$ 's over $\mathbb{Z}[t][y]$. Since the elements $\mathfrak{S}_{w}\left(\theta_{1}, \ldots, \theta_{n}\right)$ in $\widetilde{\mathcal{E}}_{n}[t]$ are linearly independent over $\mathbb{Z}[t][y]$, the homomorphism $\varphi$ is an isomorphism.

Corollary 3.1. The subalgebra of $\widetilde{\mathcal{E}}_{n, 0}$ generated by the Dunkl elements $\theta_{1}, \ldots, \theta_{n}$ over $H_{T}^{*}(\mathrm{p} t)=\mathbb{Z}\left[y_{1}, \ldots, y_{n}\right]$ is isomorphic to the $T$-equivariant cohomology ring $H_{T}^{*}\left(F l_{n}\right)$.

Proof. Let $\left(0=U_{0} \subset U_{1} \subset \cdots \subset U_{n}\right)$ be the universal flag over $F l_{n}$. Corollary 2.1 shows that the natural map $z_{i}:=-c_{1}^{T}\left(U_{i} / U_{i-1}\right) \mapsto \theta_{i}, y_{i} \mapsto y_{i}$ gives rise to a surjective homomorphism

$$
\pi: H_{T}^{*}\left(F l_{n}\right) \rightarrow \mathbb{Z}\left[y_{1}, \ldots, y_{n}\right]\left[\theta_{1}, \ldots, \theta_{n}\right] \subset \widetilde{\mathcal{E}}_{n, 0} .
$$

On the other hand, Proposition 3.2 implies that the homomorphism $\pi$ is injective.

Example 3.1. When $t=0$, our formula in Theorem 2.1 specializes to the Pieri formula in $H_{T}^{*}\left(F l_{n}\right)$ under the identification in Corollary 3.1. Let $\left\{\Omega_{w}\right\}_{w \in \mathbf{S}_{n}}$ be the Schubert basis of $H_{T}^{*}\left(F l_{n}\right)$, and let $z_{i}:=-c_{1}^{T}\left(U_{i} / U_{i-1}\right)$. Then our formula in $H_{T}^{*}\left(F l_{n}\right)$ can be written as follows:

$$
e_{k}\left(z_{1}, \ldots, z_{m}\right) \cdot \Omega_{w}=\sum_{S \subset\{1, \ldots, m\}} \prod_{i \in S} y_{w(i)} \sum_{(*)} \Omega_{w t_{i_{l} j_{l}} \cdots t_{i_{1} j_{1}}},
$$

where $(*)$ stands for the following conditions: $|S|+l=k ; 1 \leq i_{a} \leq m<j_{a} \leq n$ for $1 \leq a \leq l ; i_{1}, \ldots, i_{l}$ are distinct; $j_{1} \leq \cdots \leq j_{l}$; there exists a path $w \rightarrow w t_{i_{l} j_{l}} \rightarrow$ $w t_{i_{l} j_{l}} t_{i_{l-1} j_{l-1}} \rightarrow \cdots \rightarrow w t_{i_{l} j_{l}} \cdots t_{i_{1} j_{1}}$ in the Bruhat ordering of $\mathbf{S}_{n}$. For the cyclic permutation $[m, k]:=s_{m-k+1} s_{m-k+2} \cdots s_{m}$, the corresponding double Schubert polynomial is given as follows (see [12, Proposition 2.6.7]):

$$
\mathfrak{S}_{[m, k]}(x, y)=\sum_{j=0}^{k} e_{k-j}\left(x_{1}, \ldots, x_{m}\right) h_{j}\left(-y_{1}, \ldots,-y_{m-k+1}\right),
$$

where $h_{j}$ is the complete symmetric polynomial of degree $j$, so that the above formula gives the multiplication rule for $\Omega_{[m, k]}$ in $H_{T}^{*}\left(F l_{n}\right)$.

Now we consider an example from [14. Let $[5,5]=s_{1} s_{2} s_{3} s_{4} s_{5}$ be a permutation in $\mathbf{S}_{9}$. For $w=s_{3} s_{4} s_{5} s_{7} s_{6} s_{5}$ and $u=w t_{26} t_{16} t_{59}$, we compute the coefficient $p_{[5,5], w}^{u}$ of $\Omega_{u}$ in the expansion of $\Omega_{[5,5]} \cdot \Omega_{w}$ by using our formula. It is easily seen that there exists a unique path of length 3 from $w$ to $u$, namely,

$$
w \rightarrow w t_{59} \rightarrow w t_{59} t_{26} \rightarrow u=w t_{59} t_{26} t_{16} .
$$

Hence, the action of $\mathfrak{S}_{[m, k]}(\theta, y)$ on $\underline{w}$ under the Bruhat representation at $t=0$ is given by

$$
\begin{aligned}
\mathfrak{S}_{[m, k]}(\theta, y)(\underline{w}) & =\sum_{j=0}^{5}\left(-y_{1}\right)^{j} e_{5-j}\left(\theta_{1}, \ldots, \theta_{5}\right) \underline{w} \\
& =\left(\cdots+\left(x_{3} x_{4}-y_{1}\left(x_{3}+x_{4}\right)+y_{1}^{2}\right)[16][26][59]+\cdots\right) \underline{w} \\
& =\cdots+\left(y_{1}-y_{4}\right)\left(y_{1}-y_{6}\right) \underline{u}+\cdots,
\end{aligned}
$$


and we get $p_{[5,5], w}^{u}=\left(\alpha_{1}+\alpha_{2}+\alpha_{3}\right)\left(\alpha_{1}+\cdots+\alpha_{5}\right), \alpha_{i}=y_{i}-y_{i+1}$. This coincides with the result computed in [14, Example 4.8].

\section{§4. Quantization}

In this section we discuss a quantized version of our formula obtained in $\S 2$, which gives a multiplication rule in the quantum cohomology ring $Q H_{T}^{*}\left(F l_{n}\right)$ and a characterization of the quantum Schubert polynomials.

Definition 4.1. The algebra $\mathcal{E}_{n}^{\mathbf{q}}$ is a $\mathbb{Z}\left[q_{i j}=q_{j i} \mid 1 \leq i<j \leq n\right]$-algebra determined by the same generators and relations as in the definition of the algebra $\mathcal{E}_{n}$ except that relation (1) is replaced by

$$
(1)^{\prime} \quad[i, j]^{2}=q_{i j}
$$

for $1 \leq i<j \leq n$. The algebra $\mathcal{E}_{n}^{q}$ is defined as a $\mathbb{Z}\left[q_{1}, \ldots, q_{n-1}\right]$-algebra obtained from $\mathcal{E}_{n}^{\mathbf{q}}$ by the specialization

$$
q_{i j}= \begin{cases}q_{i} & \text { if } i=j-1, \\ 0 & \text { if } i<j-1 .\end{cases}
$$

The extension $\mathcal{E}_{n}^{\mathbf{q}}\langle R\rangle[t]$ (respectively, $\mathcal{E}_{n}^{q}\langle R\rangle[t]$ ) of the algebra $\mathcal{E}_{n}^{\mathbf{q}}$ (respectively, $\mathcal{E}_{n}^{q}$ ) is also defined by relations $(A),(B)$ and $(C)$.

In the algebra $\mathcal{E}_{n}^{\mathbf{q}}\langle R\rangle[t]$, we have a formula similar to Theorem 2.1. In order to state the formula, we need the quantum elementary symmetric polynomials $e_{k}^{\mathbf{q}}$ defined by the recursive formula

$$
\begin{gathered}
e_{k}^{\mathbf{q}}\left(X_{i} \mid i \in I \cup\{j\}\right)=e_{k}^{\mathbf{q}}\left(X_{i} \mid i \in I\right)+X_{j} e_{k-1}^{\mathbf{q}}\left(X_{i} \mid i \in I\right)+\sum_{a \in I} q_{a j} e_{k-2}^{\mathbf{q}}\left(X_{i} \mid i \in I \backslash\{a\}\right), \\
e_{0}^{\mathbf{q}}\left(X_{i} \mid i \in I\right)=1, \quad e_{k}^{\mathbf{q}}(\varnothing)=0, \quad k>0,
\end{gathered}
$$

where $I$ is a subset of $\{1, \ldots, n\}$ and $j \notin I$. The Dunkl elements $\theta_{i}=\theta_{i}^{(n)}$ in $\mathcal{E}_{n}^{\mathbf{q}}\langle R\rangle[t]$ are defined by the same formula as in the classical case.

Theorem 4.1. For $k \leq m \leq n$, we have the following formula in the algebra $\mathcal{E}_{n}^{\mathbf{q}}\langle R\rangle[t]$ :

$$
e_{k}^{\mathbf{q}}\left(\theta_{1}^{(n)}, \ldots, \theta_{m}^{(n)}\right)=\sum_{r \geq 0}(-t)^{r} N(m-k, 2 r)\left\{\sum_{S, I=\left\{i_{a}\right\},\left(j_{a}\right)} X_{S} \cdot\left[i_{1}, j_{1}\right] \cdots\left[i_{|I|}, j_{|I|}\right]\right\},
$$

where the second summation is over the triples $\left(S, I=\left\{i_{1}, \ldots, i_{|I|}\right\},\left(j_{a}\right)_{a=1}^{|I|}\right)$ such that $S \subset\{1, \ldots, m\}, I$ is a subset of $\{1, \ldots, m\} \backslash S,|I|+|S|+2 r=k, 1 \leq i_{a} \leq m<j_{a} \leq n$, and $j_{1} \leq \cdots \leq j_{|I|}$.

Proof. We use the same symbols as in the proof of Theorem 2.1. We shall show that $E_{k}(\mathcal{A})$ satisfies the recursive relation

$$
E_{k}(\mathcal{A} \cup\{j\})=E_{k}(\mathcal{A})+E_{k-1}(\mathcal{A})\left(x_{j}+\sum_{s \neq j}[j, s]\right)+\sum_{\nu \in \mathcal{A}} q_{\nu j} E_{k-2}(\mathcal{A} \backslash\{\nu\})
$$

in the algebra $\mathcal{E}_{n}^{\mathbf{q}}\langle R\rangle[t]$. All the arguments in the proof of Theorem 2.1 work, except that $C_{4}^{r}=0$; therefore,

$$
E_{k}(\mathcal{A} \cup\{j\})-E_{k}(\mathcal{A})-E_{k-1}(\mathcal{A})\left(x_{j}+\sum_{s \neq j}[j, s]\right)=-\sum_{r \geq 0}(-t)^{r} N(m-k+1,2 r) C_{4}^{r} .
$$


The following cyclic relation [13, Lemma 5.3] is fulfilled in the algebra $\mathcal{E}_{n}^{\mathbf{q}}\langle R\rangle[t]$ :

$$
\begin{aligned}
& \sum_{k=1}^{m}\left[a, i_{k}\right]\left[a, i_{k+1}\right] \cdots\left[a, i_{m}\right] \cdot\left[a, i_{1}\right] \cdots\left[a, i_{k-1}\right]\left[a, i_{k}\right] \\
& \quad=\sum_{k=1}^{m} q_{a k}\left[i_{k}, i_{k+1}\right]\left[i_{k}, i_{k+2}\right] \cdots\left[i_{k}, i_{m}\right]\left[i_{k}, i_{1}\right] \cdots\left[i_{k}, i_{k-1}\right],
\end{aligned}
$$

where $1 \leq a, i_{1}, \ldots, i_{m} \leq n$ are distinct. By using this cyclic relation, we obtain

$$
\begin{aligned}
-\sum_{r \geq 0} & (-t)^{r} N(m-k+1,2 r) C_{4}^{r} \\
& =-\sum_{r \geq 0}(-t)^{r} N(m-k+1,2 r) \sum_{S \subset \mathcal{A}} X_{S} \sum_{I_{1} \cdots I_{d} \subset_{k-1-2 r-|S|} \mathcal{A} \backslash S}\left\langle\left\langle I_{1} \mid j_{1}\right\rangle\right\rangle \cdots\left\langle\left\langle I_{d} \mid j_{d}\right\rangle\right\rangle \sum_{s \in I_{1}}[j, s] \\
& =\sum_{r \geq 0}(-t)^{r} N(m-k+1,2 r) \sum_{S \subset \mathcal{A}} X_{S} \sum_{\nu \in \mathcal{A} \backslash S} \sum_{(*)} q_{j \nu}\left\langle\left\langle I_{1} \mid j_{1}\right\rangle\right\rangle \cdots\left\langle\left\langle I_{d} \mid j_{d}\right\rangle\right\rangle\left\langle\left\langle I_{d+1} \mid \nu\right\rangle\right\rangle \\
& =\sum_{\nu \in \mathcal{A}} q_{j \nu} \sum_{r \geq 0}(-t)^{r} N((m-1)-(k-2), 2 r) \sum_{S \subset \mathcal{A} \backslash\{\nu\}} X_{S} \sum_{(*)}\left\langle\left\langle I_{1} \mid j_{1}\right\rangle\right\rangle \cdots\left\langle\left\langle I_{d+1} \mid j_{d+1}\right\rangle\right\rangle \\
& =\sum_{\nu \in \mathcal{A}} q_{j \nu} E_{k-2}(\mathcal{A} \backslash\{\nu\}),
\end{aligned}
$$

where $(*)$ means the condition $I_{1} \cdots I_{d+1} \subset_{k-2-2 r-|S|} \mathcal{A} \backslash(S \cup\{\nu\})$. This completes the proof.

The Bruhat representation for $\mathcal{E}_{n}$ is deformed to the quantum Bruhat representation for $\mathcal{E}_{n}^{q}$. Define the quantum Bruhat operator $\sigma_{i j}^{q}, i<j$, acting on $\mathbb{Z}\left[q_{1}, \ldots, q_{n-1}\right]\left\langle\mathbf{S}_{n}\right\rangle=$ $\bigoplus_{w \in \mathbf{S}_{n}} \mathbb{Z}\left[q_{1}, \ldots, q_{n-1}\right] \cdot \underline{w}$ as follows:

$$
\sigma_{i j}^{q}(\underline{w})= \begin{cases}q_{i j} \underline{w t_{i j}} & \text { if } l\left(w t_{i j}\right)=l(w)-2(j-i)+1, \\ w t_{i j} & \text { if } l\left(w t_{i j}\right)=l(w)+1, \\ 0 & \text { otherwise. }\end{cases}
$$

For $f(y) \in \mathbb{Z}\left[y_{1}, \ldots, y_{n}\right][t]$ and $w \in \mathbf{S}_{n}$, we define the $\mathbb{Z}\left[q_{1}, \ldots, q_{n-1}\right][t]$-linear operators $\tilde{\sigma}_{i j}^{q}$ by

$$
\widetilde{\sigma}_{i j}^{q}(f(y) \underline{w})=t\left(\partial_{w(i) w(j)} f(y)\right) \underline{w}+f(y) \sigma_{i j}^{q}(\underline{w}) .
$$

In the same way as in the proof of Proposition 3.1, we can check that the quantum extended Bruhat representation $[i j] \mapsto \widetilde{\sigma}_{i j}^{q}, x_{k} \mapsto \xi_{k}$ of the algebra $\mathcal{E}_{n}^{q}\langle R\rangle[t]$ is well defined.

Theorem 4.2. Let $\mathfrak{S}_{w}^{q}(x, y)$ be the quantum double Schubert polynomial corresponding to $w \in \mathbf{S}_{n}$ (see [3] and [8]). When $t=0$, we have

$$
\mathfrak{S}_{w}^{q}(\theta, y)(\underline{\mathrm{id}} .)=\underline{w}
$$

under the quantum extended Bruhat representation.

Proof. This follows from the quantum Monk formula 5 for the quantum Schubert polynomials.

Corollary 4.1. The quantum double Schubert polynomials $\mathfrak{S}_{w}^{q}(x, y)$ are characterized by the following conditions:

(1) $\left.\mathfrak{S}_{w}^{q}(x, y)\right|_{q=0}=\mathfrak{S}_{w}(x, y)$;

(2) $\mathfrak{S}_{w}^{q}(x, y)$ is a linear combination of $\mathfrak{S}_{v}(x, y)$ with $v \leq w$ over $\mathbb{Z}\left[q_{1}, \ldots, q_{n-1}\right]$;

(3) $\mathfrak{S}_{w}^{q}(\theta, y)(\underline{\mathrm{id}})=.\underline{w}$ under the quantum extended Bruhat representation at $t=0$. 
Proof. Let $\left\{P_{w}(x, y)\right\}_{w \in \mathbf{S}_{n}}$ be a family of polynomials that satisfy the above properties (1), (2) and (3) of the quantum Schubert polynomials. We shall show that $P_{w}(x, y)=\mathfrak{S}_{w}^{q}(x, y)$ for all $w \in \mathbf{S}_{n}$. Let $S=1+\left(q_{1}, \ldots, q_{n-1}\right)$ be a multiplicative system of $\mathbb{Z}\left[q_{1}, \ldots, q_{n-1}\right]$ consisting of polynomials of the form $1+\sum_{i=1}^{n-1} q_{i} a_{i}(q)$, $a_{i}(q) \in \mathbb{Z}\left[q_{1}, \ldots, q_{n-1}\right]$. Properties (1) and (2) imply that

$$
\mathfrak{S}_{w}^{q}(x, y)=\sum_{v \leq w} b_{w}^{v}(q) \mathfrak{S}_{v}(x, y), \quad b_{w}^{v}(q) \in \mathbb{Z}\left[q_{1}, \ldots, q_{n-1}\right], \quad b_{w}^{w}(q) \in S .
$$

Then it is easy to check that

$$
\mathfrak{S}_{w}(x, y)=\sum_{v \leq w} c_{w}^{v}(q) \mathfrak{S}_{v}^{q}(x, y), \quad c_{w}^{v}(q) \in S^{-1} \mathbb{Z}\left[q_{1}, \ldots, q_{n-1}\right],
$$

by induction on the Bruhat ordering. Similarly, we also have

$$
\mathfrak{S}_{w}(x, y)=\sum_{v \leq w} d_{w}^{v}(q) P_{v}(x, y), \quad d_{w}^{v}(q) \in S^{-1} \mathbb{Z}\left[q_{1}, \ldots, q_{n-1}\right] .
$$

Hence,

$$
\sum_{v \leq w}\left(c_{w}^{v}(q) \mathfrak{S}_{v}^{q}(x, y)-d_{w}^{v}(q) P_{v}(x, y)\right)=0
$$

for all $w \in \mathbf{S}_{n}$. From property (3), we obtain

$$
\sum_{v \leq w}\left(c_{w}^{v}(q)-d_{w}^{v}(q)\right) \underline{v}=0
$$

for all $w \in \mathbf{S}_{n}$, whence $c_{w}^{v}(q)=d_{w}^{v}(q)$ for all $w \in \mathbf{S}_{n}$ and $v \leq w$. Using these identities, we can conclude that $P_{w}(x, y)=\mathfrak{S}_{w}^{q}(x, y)$, again by induction on the Bruhat ordering.

\section{§5. Nichols-Woronowicz MOdel}

The model of the equivariant cohomology ring $H_{T}^{*}\left(F l_{n}\right)$ in the algebra $\widetilde{\mathcal{E}}_{n}$ has a natural interpretation in terms of the Nichols-Woronowicz algebra. The Nichols-Woronowicz approach leads us to the uniform construction for arbitrary root systems. For the definition of the Nichols-Woronowicz algebra, see, e.g., 11, 2, 11] and [16.

We denote by $\mathcal{B}_{W}$ the Nichols-Woronowicz algebra associated with the Yetter-Drinfeld module

$$
V=\bigoplus_{\alpha \in \Delta} \mathbf{R}[\alpha] /([\alpha]+[-\alpha])
$$

over the finite Coxeter group $W$ of the root system $\Delta$. Let $\mathfrak{h}$ be the reflection representation of $W$ and $R=S_{y m \mathfrak{h}}$ the ring of polynomial functions on $\mathfrak{h}$. Consider the extension $\mathcal{B}_{W}\langle R\rangle[t]$ of the algebra $\mathcal{B}_{W}$ by the polynomial ring $R[t]$ defined by the commutation relation

$$
[\alpha] x=s_{\alpha}(x)[\alpha]+t(x, \alpha) \text { for } \quad x \in \mathfrak{h}^{*} .
$$

Definition 5.1. We define the $R$-algebra $\widetilde{\mathcal{B}}_{W}$ by

$$
\widetilde{\mathcal{B}}_{W}=\mathcal{B}_{W}\langle R\rangle[t] \otimes_{R^{W}} R
$$

Choosing $W$-invariant constants $\left(c_{\alpha}\right)_{\alpha}$, we consider a linear map $\mu: \mathfrak{h}^{*} \rightarrow \widetilde{\mathcal{B}}_{W}$ defined as

$$
\mu(x)=x+\sum_{\alpha \in \Delta_{+}} c_{\alpha}(x, \alpha)[\alpha]
$$

for $x \in \mathfrak{h}^{*}$.

Proposition 5.1. $[\mu(x), \mu(y)]=0, x, y \in \mathfrak{h}^{*}$. 
Proof. Let $\mu_{0}(x):=\sum_{\alpha \in \Delta_{+}} c_{\alpha}(x, \alpha)[\alpha]$. Here we may normalize the length of the roots to have $(\alpha, \alpha)=1, \alpha \in \Delta$. The commutativity $\left[\mu_{0}(x), \mu_{0}(y)\right]=0$ was shown in [2]. The commutativity between $\mu(x)$ and $\mu(y)$ follows from

$$
\begin{aligned}
\mu_{0}(x) y & +x \mu_{0}(y) \\
= & \sum_{\alpha \in \Delta_{+}} c_{\alpha}(x, \alpha) s_{\alpha}(y)[\alpha]+t \sum_{\alpha \in \Delta_{+}} c_{\alpha}(x, \alpha)(y, \alpha)+\sum_{\alpha \in \Delta_{+}} c_{\alpha}(y, \alpha) x[\alpha] \\
= & \sum_{\alpha \in \Delta_{+}} c_{\alpha}(x, \alpha) y[\alpha]-\sum_{\alpha \in \Delta_{+}} 2 c_{\alpha}(x, \alpha)(y, \alpha) \alpha[\alpha] \\
& +t \sum_{\alpha \in \Delta_{+}} c_{\alpha}(x, \alpha)(y, \alpha)+\sum_{\alpha \in \Delta_{+}} c_{\alpha}(y, \alpha) x[\alpha] \\
= & \sum_{\alpha \in \Delta_{+}} c_{\alpha}(x, \alpha) y[\alpha]+t \sum_{\alpha \in \Delta_{+}} c_{\alpha}(x, \alpha)(y, \alpha)+\sum_{\alpha \in \Delta_{+}} c_{\alpha}(y, \alpha) s_{\alpha}(x)[\alpha] \\
= & y \mu_{0}(x)+\mu_{0}(y) x .
\end{aligned}
$$

Proposition 5.1 shows that the linear map $\mu$ extends to a homomorphism of algebras

$$
\mu: R \rightarrow \mathcal{B}_{W}\langle R\rangle[t] .
$$

Denote by $\widetilde{\mu}$ the composition of the homomorphisms

$$
R \otimes_{\mathbf{R}} R \stackrel{\mu \otimes 1}{\rightarrow} \mathcal{B}_{W}\langle R\rangle[t] \otimes_{\mathbf{R}} R \rightarrow \widetilde{\mathcal{B}}_{W} .
$$

In Theorem 5.1 below we shall show that the image of the algebra homomorphism $\widetilde{\mu}$ at $t=0$ is isomorphic to the algebra $R \otimes_{R^{W}} R$. The proof is based on the correspondence between the twisted derivation $D_{\alpha}$ and the divided difference operator $\partial_{\alpha}:=\left(1-s_{\alpha}\right) / \alpha$, which acts on the first tensor component of $R \otimes_{R^{W}} R$ and extends linearly with respect to the second tensor component. We define the operator $D_{\alpha}$ as the twisted derivation on $\widetilde{\mathcal{B}}_{W}$ determined by the conditions

(1) $D_{\alpha}(x)=0$ for $x \in R$,

(2) $D_{\alpha}([\beta])=\delta_{\alpha, \beta}$ for $\alpha, \beta \in \Delta_{+}$,

(3) $D_{\alpha}(f g)=D_{\alpha}(f) g+s_{\alpha}(f) D_{\alpha}(g)$.

The operator $D_{\alpha}$ is linear with respect to $R$ on the second component.

\section{Proposition 5.2.}

$$
\bigcap_{\alpha \in \Delta_{+}} \operatorname{Ker}\left(D_{\alpha}\right)=R[t] \otimes_{R^{W}} R .
$$

Proof. Since $\mathcal{B}_{W}\langle R\rangle[t]$ is isomorphic to $R[t] \otimes_{\mathbf{R}} \mathcal{B}_{W}$ as a right $\mathcal{B}_{W}$-module, any element $\omega \in \mathcal{B}_{W}\langle R\rangle[t]$ can be written as

$$
\omega=f_{1} \varphi_{1}+\cdots+f_{k} \varphi_{k},
$$

where $f_{1}, \ldots, f_{k} \in R[t]$ are linearly independent, and $\varphi_{1}, \ldots, \varphi_{k} \in \mathcal{B}_{W}$. We have

$$
D_{\alpha}(\omega)=s_{\alpha}\left(f_{1}\right) D_{\alpha}\left(\varphi_{1}\right)+\cdots+s_{\alpha}\left(f_{k}\right) D_{\alpha}\left(\varphi_{k}\right)
$$

by the twisted Leibniz rule. If $D_{\alpha}(\omega)=0$, we have $D_{\alpha}\left(\varphi_{1}\right)=\cdots=D_{\alpha}\left(\varphi_{k}\right)=0$. Hence, if $\omega \in \bigcap_{\alpha \in \Delta_{+}} \operatorname{Ker}\left(D_{\alpha}\right)$, then $\varphi_{i}$ belongs to the homogeneous part $\mathcal{B}_{W}^{0} \cong \mathbf{R}$ of degree zero for $i=1, \ldots, k$. This means that $\omega \in R[t]$.

Proposition 5.3. For $x \in R \otimes_{\mathbf{R}} R$,

$$
D_{\alpha}(\widetilde{\mu}(x))=c_{\alpha} \widetilde{\mu}\left(\partial_{\alpha}(x)\right) .
$$


Proof. If $x=\beta \otimes 1, \beta \in \Delta$, we can check the relation

$$
D_{\alpha}(\widetilde{\mu}(\beta \otimes 1))=c_{\alpha}(\beta, \alpha)=c_{\alpha} \widetilde{\mu}\left(\partial_{\alpha}(\beta)\right) .
$$

Hence, we have $D_{\alpha}(\widetilde{\mu}(x))=c_{\alpha} \widetilde{\mu}\left(\partial_{\alpha}(x)\right)$ for $x \in \mathfrak{h}^{*} \otimes R$. On the other hand, the two sides satisfy the same twisted Leibniz rule, whence it follows that $D_{\alpha}(\widetilde{\mu}(x))=c_{\alpha} \widetilde{\mu}\left(\partial_{\alpha}(x)\right)$ for $x \in R \otimes R$.

Theorem 5.1. If $t=0$ and the constants $\left(c_{\alpha}\right)_{\alpha}$ are generic, the image of the homomorphism $\widetilde{\mu}$ is isomorphic to the algebra $R \otimes_{R^{W}} R$. In particular, when $W$ is the Weyl group, it is isomorphic to the $T$-equivariant cohomology ring $H_{T}^{*}(G / B)$ of the corresponding flag variety $G / B$.

Proof. If $x \in R^{W} \otimes_{\mathbf{R}} R$, we have $D_{\alpha}(\widetilde{\mu}(x))=0$ for every $\alpha \in \Delta_{+}$by Proposition 5.3. This implies, by Proposition 5.2, that $\widetilde{\mu}(x) \in R^{W} \otimes_{R^{W}} R$. When $t=0, \widetilde{\mu}(x)$ coincides with the element of $R$ obtained by replacing all the symbols $[\alpha]$ with zero in $\widetilde{\mu}(x)$. Hence, the homomorphism $\widetilde{\mu}$ factors through $R \otimes_{R^{W}} R \rightarrow \widetilde{\mathcal{B}}_{W}$.

We take a reduced decomposition $w=s_{\alpha_{i_{1}}} \cdots s_{\alpha_{i_{l}}}$ of an element $w \in W$. Then the operator $\partial_{w}:=\partial_{\alpha_{i_{1}}} \cdots \partial_{\alpha_{i_{l}}}$ is independent of the choice of reduced decompositions, thanks to the Coxeter relation. We also define $D_{w}:=D_{\alpha_{i_{1}}} \cdots D_{\alpha_{i_{l}}}$. We introduce a family $\left\{X_{w}\right\}_{w \in W}$ of polynomials by $X_{w_{0}}:=|W|^{-1} \prod_{\alpha \in \Delta_{+}} \alpha$ and $X_{w}:=\partial_{w^{-1} w_{0}} X_{w_{0}}$. The family $\left\{X_{w}\right\}_{w \in W}$ gives a linear basis of the coinvariant algebra $R_{W}$. We can see that

$$
C T\left(\partial_{w} X_{v}\right)= \begin{cases}1 & \text { if } w=v \\ 0 & \text { otherwise }\end{cases}
$$

where $C T$ stands for the part of degree zero. Since a linear basis $\left\{X_{w}\right\}_{w \in W}$ of the coinvariant algebra of $W$ gives an $R^{W}$-basis of $R$, and $D_{w} \widetilde{\mu}\left(X_{v} \otimes 1\right)=c_{\alpha_{i_{1}}} \cdots c_{\alpha_{i_{l}}} \widetilde{\mu}\left(\partial_{w} X_{v} \otimes 1\right)$, it is easy to check that $R \otimes_{R^{W}} R \rightarrow \widetilde{\mathcal{B}}_{W}$ is injective.

Remark 5.1. Our construction is not a straightforward application of the functor $(-) \otimes_{R^{W}}$ $R$ to the one given in [2] even when $t=0$. In fact, the defining relations of the algebra $\mathcal{B}_{W}\langle R\rangle[t]$ involve a nontrivial commutation relation

$$
[\alpha] x=s_{\alpha}(x)[\alpha]+t(x, \alpha) .
$$

Moreover, in the formula

$$
\mu(x)=x+\sum_{\alpha \in \Delta_{+}} c_{\alpha}(x, \alpha)[\alpha],
$$

the first term on the right-hand side does not occur in the nonequivariant case.

\section{REFERENCES}

[1] N. Andruskiewitsch and H.-J. Schneider, Pointed Hopf algebras, New Directions in Hopf Algebras, Math. Sci. Res. Inst. Publ., vol. 43, Cambridge Univ. Press, Cambridge, 2002, pp. 1-68. MR1913436 (2003e:16043)

[2] Y. Bazlov, Nichols-Woronowicz algebra model for Schubert calculus on Coxeter groups, J. Algebra 297 (2006), no. 2, 372-399. MR2209265 (2007b:20083)

[3] I. Ciocan-Fontanine and W. Fulton, Quantum double Schubert polynomials, Inst. Mittag-Leffler Report no. 6, 1996-1997.

[4] C. Dunkl, Harmonic polynomials and peak sets of reflection groups, Geom. Dedicata 32 (1989), 157-171. MR:1029672 (91h:51015)

[5] S. Fomin, S. Gelfand, and A. Postnikov, Quantum Schubert polynomials, J. Amer. Math. Soc. 10 (1997), 565-596. MR1431829 (98d:14063)

[6] S. Fomin and A. N. Kirillov, Quadratic algebras, Dunkl elements, and Schubert calculus, Advances in Geometry (J.-L. Brylinski, R. Brylinski, V. Nistor, B. Tsygan, and P. Xu, eds.), Progr. Math., vol. 172, Birkhäuser Boston, Boston, MA, 1999, pp. 147-182. MR1667680 (2001a:05152) 
[7] A. N. Kirillov, On some quadratic algebras. II, Preprint.

[8] A. N. Kirillov and T. Maeno, Quantum double Schubert polynomials, quantum Schubert polynomials and Vafa-Intriligator formula, Discrete Math. 217 (2000), 191-223. MR 1766267 (2001f:05161)

[9] _ Noncommutative algebras related with Schubert calculus on Coxeter groups, European J. Combin. 25 (2004), 1301-1325. MR2095483 (2005j:05097)

[10] A. Lascoux and M.-P. Schützenberger, Polynômes de Schubert, C. R. Acad. Sci. Paris Ser. I Math. 294 (1982), 447-450. MR 0660739 (83e:14039)

[11] S. Majid, Free braided differential calculus, braided binomial theorem, and the braided exponential map, J. Math. Phys. 34 (1993), 4843-4856. MR1235979(94i:58013)

[12] L. Manivel, Symmetric functions, Schubert polynomials and degeneracy loci, SMF/AMS Texts and Monogr., vol. 6, Amer. Math. Soc., Providence, RI, 2001. MR1852463 (2002h:05161)

[13] A. Postnikov, On a quantum version of Pieri's formula, Advances in Geometry (J.-L. Brylinski, R. Brylinski, V. Nistor, B. Tsygan, and P. Xu, eds.), Progr. Math., vol. 172, Birkhäuser Boston, Boston, MA, 1999, pp. 371-383. MR1667687 (99m:14096)

[14] S. Robinson, A Pieri type formula for $H_{T}^{*}\left(\mathrm{SL}_{n}(\mathbf{C}) / \mathbf{B}\right)$, J. Algebra 249 (2002), 38-58. MR.1887984 (2003b:14065)

[15] S. Veigneau, Calcul symbolique et calcul distribué en combinatoire algébrique, Thèse, Univ. Marnela-Valée, 1996.

[16] S. L. Woronowicz, Differential calculus on compact matrix pseudogroups (quantum groups), Comm. Math. Phys. 122 (1989), 125-170. MR0994499 (90g:58010)

Research Institute for Mathematical Sciences, Kyoto University, Sakyo-ku, Kyoto 6068502, JAPAN

E-mail address: kirillov@kurims.kyoto-u.ac.jp

Department of Electrical Engineering, Kyoto University, Sakyo-ku, Kyoto 606-8501, JAPAN

E-mail address: maeno@kuee.kyoto-u.ac.jp

Received 15/JAN/2010

Originally published in English 